\title{
Supporting Collaboration with Non-Literate Forest Communities in the Congo-Basin
}

\author{
Michalis Vitos ${ }^{1}$ \\ Gillian Conquest ${ }^{1}$ \\ Julia Altenbuchner ${ }^{1}$ \\ Matthias Stevens ${ }^{1,2}$ \\ Jerome Lewis ${ }^{1}$ \\ Muki Haklay ${ }^{1}$ \\ 1 Extreme Citizen Science (ExCiteS) group, University College London, UK \\ \{ michalis.vitos.11, julia.altenbuchner.11, m.stevens, g.conquest.11, jerome.lewis, m.haklay \}@ucl.ac.uk \\ 2 iMinds Living Labs, IMEC, Ghent, Belgium, matthias.stevens@iminds.be
}

\begin{abstract}
Providing indigenous communities with ICT tools and methods for collecting and sharing their Traditional Ecological Knowledge is increasingly recognised as an avenue for improvements in environmental governance and socialenvironmental justice. In this paper we show how we carried out a usability engineering effort in the "wild" context of the Congolese rainforest - designing, evaluating and iteratively improving novel collaborative data collection interfaces for non-literate forest communities that can subsequently be used to facilitate communication and information sharing with logging companies. Working in this context necessitates adopting a thoroughly flexible approach to the design, development, introduction and evaluation of technology and the modes of interaction it offers. We show that we have improved participant accuracy from about $75 \%$ towards $95 \%$ and provide a set of guidelines for designing and evaluating ICT solutions in "extreme circumstances" - which hold lessons for CSCW, HCI and ICT4D practitioners dealing with similar challenges.
\end{abstract}

\section{Author Keywords}

HCI4D; ICT4D; CSCW; Usability experiments; Research in the wild; Audio interfaces; Physical interfaces; Participatory mapping; Natural resource management.

\section{ACM Classification Keywords}

H.5.2 User Interface: Auditory feedback, Evaluation/methodology, User-centered design, Interaction styles; H.5.m. Information Interfaces and Presentation (e.g. HCI): Miscellaneous

\section{INTRODUCTION}

"I have never used a mobile phone in my life and I am so happy that it was so easy" commented a 39 year-old Mbendjele woman after trying the Tap\&Map mobile app for the first time. She lives in a small forest community in the Congo

Permission to make digital or hard copies of part or all of this work for personal or classroom use is granted without fee provided that copies are not made or distributed for profit or commercial advantage and that copies bear this notice and the full citation on the first page. Copyrights for third-party components of this work must be honored. For all other uses, contact the owner/author(s).

CSCW '17 February 25 - March 01, 2017, Portland, OR, USA

(C) 2017 Copyright held by the owner/author(s).

ACM ISBN 978-1-4503-4335-0/17/03

DOI: http://dx.doi.org/10.1145/2998181.2998242
Basin, has never had the opportunity to receive a formal education or to interact with modern technology, and yet she is collecting digital, geographical information through a smartphone. She is mapping nearby trees that are important to her people, with the aim of gathering evidence to protect these resources. We observed and evaluated how she and her peers used our Sapelli application, developed specifically for this context, and explored how novel ways of device interaction like Tap\&Map can assist non-literate people to record and communicate their local knowledge to local decision makers.

Sustainable management of natural resources is one of the fundamental challenges of our age. Local and indigenous communities often possess unique knowledge about the natural resources on which their livelihoods depend. This Traditional Ecological Knowledge is increasingly recognised as critical for sustaining these resources [5, 24, 26]. Recent technological developments, and growing acceptance of different forms of knowledge, mean that participatory citizen science is seen as a promising solution to achieve long-term management of key environments with greater respect for, and an active role accorded to, local communities $[7,16]$. In addition, "on the ground" data collection can provide a "triangulation" of the reality and provide ground truth [65].

As part of an interdisciplinary research group, we focus on facilitating collaboration and communication between local, forest communities and relevant stakeholders through ICT. Our goal is to design, develop and evaluate tools that enable community members to collaborate with their peers to document local environmental conditions and knowledge, and share that information with relevant outsiders. More specifically, we seek to enable vulnerable communities to conduct their own environmental monitoring or mapping using mobile devices, with the purpose of asserting their rights, managing responses to ecological changes, or initiating a communication channel with policy makers and other stakeholders. The case studies presented in this paper took place in the remote and challenging environment of the Congolese rainforest, where we collaborated with forest communities and local intermediaries such as non-governmental organizations (NGOs), logging companies and national parks.

Research indicates that usability i.e. perceived usefulness and ease-of-use, and satisfaction play the leading role in the acceptance and adoption of a technology $[13,4]$. In the case of collaborative data collection, the usability of the suggested 
ICT solution can negatively influence the validity of the collected data and thus impact the success of an (e.g.) environmental monitoring project. Hence, this paper focuses on the usability evaluation and improvement of our CSCW tools, in order to facilitate the acceptance of the technology.

Collaborating, however, with forest communities, who are generally non-literate and lack prior exposure to ICT, introduces a range of socio-cultural, practical, methodological and interaction challenges $[25,58,64,35]$ which can only be met through participatory and culturally-informed methods of community engagement, interaction design and evaluation. Hence it is important to be working "in the wild" [50, 47], or "on the ground" [65], as much as possible. However, working in these remote and extreme places presents a number of logistical, organisational, legal, financial and security-related challenges [64] which can only be met with the cooperation of locally-situated intermediaries and which often severely limit the time we can actually spend with user communities. Consequently we find ourselves adopting a creative and agile approach to usability engineering [51] and evaluation which also can provide valuable lessons to $\mathrm{CSCW}$ and $\mathrm{HCI}$ researchers working in similarly challenging or constrained contexts.

In this paper we outline how we apply an action research [46] methodology to introduce, evaluate and adapt ICT systems with communities with little or no formal education or prior exposure to technology, and whose socio-cultural background and understanding may differ wildly from those within which such systems are usually designed. In the next section, we look at related work, while section Sapelli platform describes our mobile data collection platform. Next, section Cоттиnity Engagement in the Wild describes the process of codesigning and introducing Sapelli surveys. This provides the background to section Usability Engineering in the Wild, the primary focus of our paper. Here we describe the wide range of practical and interaction challenges encountered working in the "real wild" setting of the Congo Basin rainforest and discuss the iterative approach we followed to design and evaluate solutions to these issues. We propose four interface designs that may be suitable in this context and describe a series of experiments to test the relative usability of three of them. Finally, in the Discussion section, we provide a set of guidelines for designing and evaluating ICT solutions in these or similar "extreme circumstances", and assess the conditions under which the proposed designs may be more or less applicable.

\section{RELATED WORK}

According to UNESCO's Institute for Statistics, 774 millions worldwide are unable to read or write [60]. While this roughly translates to 1 adult in 5 being illiterate, HCI literature suggests that language and literacy are major barriers to the use of technology $[6,9,11,27,28,36,29]$, and proposes the use of interfaces free of textual and numerical information $[6,18,19,27,29,33,39,53,63]$. A large and growing crop of literature on HCI for non-literate and illiterate users pays particular attention on a) improving the usability of current mobile or web applications such as the phone book [6,
$11,27]$; b) improving participants' access to digital information, often relating to health $[20,54]$ or agriculture $[44$, $43,62]$; and c) providing finance services to participants [41, 36]. Researchers experiment with colours, symbols and icons to avoid textual user interfaces (UIs) [6, 27, 38], while others propose the use of rich media [36] or spoken dialogue systems [44, 54].

In terms of data collection, digital forms offer more efficient and convenient data collection with fewer errors compared to paper-based surveys [31, 45, 56, 59, 64]. Hence, in the context of humanitarian aid, development or conservation projects in the developing world, mobile data collection platforms running on PDAs or mobile phones are gaining success [64]. The first data collection platforms targeted handheld computers or PDAs [30], with CyberTracker as the prime example [12]. CyberTracker was designed for non-literate animal trackers to record observations $[21,55]$ and evolved into a generic data collection tool used in conservation projects [3, $15,17,42]$. In a similar vain, Lewis [32] describes the collaboration between a forest tribe and the logging company Congolaise Industrielle des Bois (CIB) to provide the community with PDA devices running bespoke software with an interface consisting of pictorial icons, to record the locations of important resources. Nowadays both platforms are outdated, primarily because they relied on expensive and equally outdated devices that lack the processing power and built-in sensors of today's smartphones [64].

The next generation of tools targeted mobile phones and smartphones, with Open Data Kit (ODK) $[2,21]$ and EpiCollect [1] as successful examples. Both platforms facilitate form-based data collection, where forms are uploaded in a centralised database, and offer tools to visualise and analyse the results. However, both tools were designed for literate users with interfaces heavily depending on textual interactions. Vitos et al. [63, 64] modified ODK to enable non-literate community participants to capture information on poaching activities and report them to local authorities. However, the authors suggest that modifying and maintaining ODK does not mitigate the complexity of ODK forms.

\section{SAPELLI PLATFORM}

Our work builds on all the above literature and in 2012 we drew up a list of requirements for a generalised, smartphonebased data collection platform to be used across our collaborations with forest communities based in the Congo Basin. The main requirements were: usability for non-literate people through the use of pictorial elements, rather than text or numbers; a flexible means to define and modify surveys, ideally without requiring programming experience; the ability to function offline; autonomous data synchronisation that can operate via SMS as well as the Internet; and last but not least the app had to be free. We reviewed several existing mobile data collection and surveying platforms. This included systems designed for specific use cases - for instance, CyberTracker [3] targets park rangers working in wildlife conservation - as well as tools with a broader scope - like EpiCollect [1] and ODK [2]. At the time none of these platforms met all the requirements and so we set out to develop our 

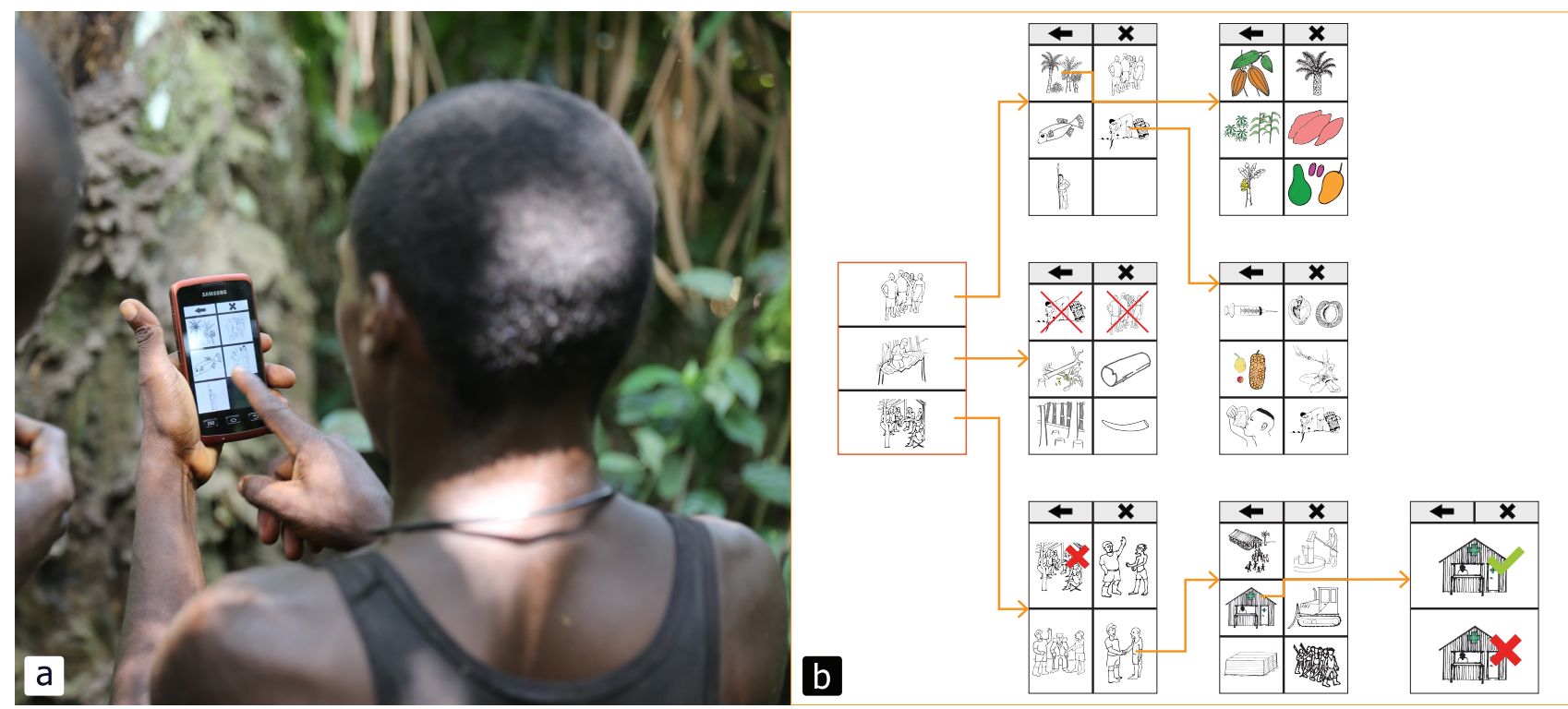

Figure 1. Sapelli platform: a) participant using the app b) decision tree designed in collaboration with Forests Monitor, CAGDF and local communities.

own. We named it Sapelli after the endangered sapelli tree (Entandrophragma cylindricum), which grows in the Congo Basin rainforest and is valued equally by indigenous locals, for whom it is a source of edible caterpillars, and by loggers, for whom it is a source of lucrative timber [57, 35].

Sapelli runs on Android phones and tablets, and is designed to be generic. The platform is intended to enable communities with varying needs and abilities to engage in mobile data collection - often, but not necessarily, across language or literacy barriers - in a wide variety of scenarios and contexts - potentially beyond those of our own projects. In fact, nothing about the Sapelli app is specific to trees or forests. Instead, much like a Web browser renders any content described as HTML, the app executes surveys described in a bespoke XML-based language which is designed to be highly readable and simple enough for anyone with basic computer skills, but no prior programming experience, to learn in a few hours.

A typical Sapelli survey takes the form of a pictorial decision tree. The tree represents a question with a predefined set of answers organised in a hierarchical structure. The leaves represent the most specific answers or classifications, while the in-between nodes represent categories or groups that lead to these final answers. Users navigate the decision space by repeatedly "tapping" images to select child nodes until they reach a leaf node (Figure 1b). Sapelli supports multiple decision trees in sequence and thus it can collect answers for multiple questions.

\section{COMMUNITY ENGAGEMENT IN THE WILD}

The approach for engaging with communities and introducing our tools is adapted from projects conducted previously in the Congo Basin [32] and is always refined in response to local conditions.
Partnerships with intermediary actors play an important role if participatory monitoring projects are to be funded and managed sustainably. It is therefore often necessary to begin by establishing relationships with intermediaries who have a sustained local presence, sufficient expertise in local conditions, and who are trusted by local communities. Such partnerships work to lend legitimacy to a project from the point of view of participants and other local stakeholders.

Upon arrival in a community we always begin with a thorough process of free, prior, and informed consent (FPIC) [34], introducing ourselves first to the local chiefs or authorities, and then to a wider assembly of the local population. Once we have received consent, we inclusively engage community members in a participatory exercise. To ensure that the gathered sample is representative, we seek to involve both adult males and females, of various ages and different ethnicities.

Since, we follow a bottom-up and AR approach, we enable communities to decide the project scope and design the project's details. After the initial FPIC process, if the community expresses an interest in participating, the next phase is an exercise in co-creation and iterative participatory design. Having understood the purpose of our collaboration (e.g. collection of data to monitor logging activities), participants now contribute to developing the data collection interface. The first step is to define the types of information to be collected. Working with a prototype, the key measurements, environmental parameters or local observations to be made are discussed. Participants comment on their ability and willingness to provide the information (e.g. observations of illegal activities can have consequences), and whether they consider it relevant to do so. We encourage people to suggest other or additional types of information they consider important. In this conceptual phase, we endeavour to also include representatives from local stakeholders (i.e. local NGO monitoring logging concession) to comment on what it is also important 
for them to be captured. This phase is spanned over many days and different communities, and during this discussion a commonly understood set of concepts, categorisation and representations (e.g. terminology or iconography) are established to structure collecting and visualising of data later on. We refrain from introducing technology during this conceptual phase so as not to distract or confuse people.

After the conceptual stage, there is the need for an ICT solution to enable communities capture the measurements that they decided are important for collection. Thus the need to develop Sapelli was born, since none of the existing ICT solutions offered pictorial-only UIs; flexible means to define and modify surveys on the spot while discussing the project with communities; the ability to function offline and autonomous data synchronisation.

When introducing Sapelli, and since pictorial decision trees play a central role in our work, we firstly engage the crowd in training exercises, where we introduce them to the pictorial icons, printed on large flashcards (Figure 3a). To ensure the icons are clearly understood, we ask the assembled crowd to guess what each image represents and whether it is considered relevant to them - this has the added advantage of making the exercise fun, participatory and accessible. We take notes concerning unclear icons, missing situations and suggested alterations or additions. We then update the decision tree before visiting the next community, meaning all feedback is incorporated into a process of iterative design. Next, we introduce the phones and demonstrate how to navigate the interface. Once people seem comfortable with the way of "tapping" the images and moving between screens, we ask them to find specific icons in order to familiarise themselves with the structure of the decision tree. It is vital to contextualise these activities to make sure people understand what the system is for and why it is relevant to them, so once we have trained a group of people we ask small teams of men and women to take the phones into the surrounding area and map some of their nearby resources. Throughout the process we continue listening for comments and ask for suggestions for possible improvements. All participant interactions with the devices are video-recorded for later reference.

\section{USABILITY ENGINEERING IN THE WILD}

During the development and evaluation of Sapelli, we followed a user-centered design (UCD) approach. Typically UCD is characterised by multiple experimental iterations with the end users, however, in our case studies access to participants is restricted by a series of challenges: distance, costs and logistics for organising a field trip and getting in contact with participants; stakeholder expectations which do not always match with our research priorities; cultural barriers; and time constraints. To mitigate these, we have adopted a flexible approach for the design and evaluation of the platform "in the wild", including two major lengthy field visits in the last 2 years, where we had multiple sub-iterations with different communities, and on-the-spot creation and evaluation of new software features and interaction prototypes.

In the first field trip our goal was to investigate the technical feasibility of the project and the appropriateness of pictorial decision trees in terms of usability, effectiveness and user experience. As explained in the Pictorial decision trees section, during our field visit we identified a series of interaction challenges, as well as cultural differences in terms of evaluating software and conducting structured experiments.

For our second field trip, we drafted strategies for conducting more successful usability experiments while in the field, and we designed and implemented two new features in our Sapelli platform as potential solutions to the identified usability issues. The first one was Audio feedback, a feature offering audio guidance to participants while navigating the pictorial decision tree, as described in Audio feedback section. The second feature was Animated transitions between different nodes of the decision tree to help participants perceive the underlying structure and create visual and mental links between the different screens.

Our limited time in the field (e.g. 4-6 weeks) requires us to adopt a flexible and agile approach. Based on our results with pictorial decision trees and audio feedback, we decided to skip the planned experiments with animated screen transitions and instead started to explore physical, tangible interfaces as a novel means to interact with Sapelli, potentially resulting in increased participant performance and satisfaction, as described in the section Exploring physical interfaces.

\section{Pictorial decision trees}

In 2013, we collaborated with the international NGO Forests Monitor and Cercle d'Appui à la Gestion Durable des Forêts [Circle of Support for Sustainable Forest Management] (CAGDF), a forestry sector watchdog in Republic of the Congo (RoC), to prototype tools for local communities to monitor the socio-economic impacts of logging activities. The resulting application, based on our Sapelli platform was intended to enable participants to map their resources and feedback on the behaviour of logging activities to CAGDF. They could then assist communities to seek redress for violations of the social aspects of forestry laws.

We spent a total of 6 weeks in northern RoC, where we visited eight different communities in the Sangha and Likouala regions. As noted above, initially we collaborated with the communities to design a decision tree and a set of icons that would be parsed by Sapelli. When the decision tree was stable (i.e. communities requested no additional changes), we evaluated Sapelli to investigate the technical feasibility of the project on the one hand, and study the usability of Sapelli and the devices on the other. During this period we had the opportunity to introduce Sapelli, through training sessions and mapping exercises, to 276 participants (146 males, 130 females). Some technical challenges such as battery life, or inconsistent performance of local cellular networks were encountered, but as the focus of this paper is on the usability of the platform, it is necessary to list the interaction challenges that were identified by observing community members using the software, as well as the cultural hurdles encountered when conducting usability experiments in this context. 


\section{Interaction challenges}

Interaction with the devices, specifically the touch-screens, proved challenging and frustrating for some people. Participants were unsure of how long they needed to press on an icon and tended to perform a long-click rather than a short tap. In other cases participants assumed that their tap was not registered, and tapped twice or more on the same spot, resulting in them navigating deeper within the decision tree by accident. We tried to tackle this on-the-spot by introducing a short waiting animation to show that a tap has been successful and the new screen will appear shortly.

Since the icons were co-designed with the community, the majority of them were easily recognised, but the interpretation of some icons caused major challenges. While most icons were intended to be interpreted literally (e.g. a banana tree means just that), others were representing categories, which proved to be more challenging. In some cases these categorical icons occurred as a final choice, but more often they were used at the top or intermediary levels of the decision tree. In the former case this usually meant there was no need for further detail. For example, a drawing of a particular kind of wild fruit might represent any wild fruit - without a need to record which kind. In the latter case, the icon typically summarised a set of possibilities that the user could choose from in the next screen. For instance, a drawing of a specific game species might represent the concept of hunting, and tapping it would then take the user to a screen offering a choice between multiple icons, each representing a particular game species. Often this also meant the same icon occurred as both an intermediate category and as a specific final answer. During the training and mapping exercises it was observed that both kinds of categorical icon were often interpreted literally and caused confusion, especially when there was no differentiation between a final leaf icon and an intermediate one.

Interface navigation and the understanding of certain icons were further hampered by the fact that we used symbolic or metaphorical conventions (e.g. arrows, crosses, ticks, the use of green and red to respectively signify positive and negative connotations) which were unknown or interpreted differently. Many participants seemed to have difficulty understanding the overall hierarchical structure and how to navigate through it using forward or backward steps. These results are aligned with research undertaken by Medhi et al. [37] in Bangalore, who found that level of formal education is positively correlated with cognitive skills such as conceptual abstraction and categorisation, and thus with the ability to apply these skills when navigating hierarchical interfaces, even when they are text-free. This may also explain the above-mentioned difficulties users had when dealing with icons that represent categories, even when occurring as leaves.

\section{Usability Experiments}

Towards the end of our field trip, on top of observing participants, we attempted to undertake structured usability experiments by exposing users to a set of predefined scenario tasks to evaluate their efficiency, accuracy and recall. Participants were presented with hypothetical scenarios and were asked to use the application to take action depending on the scenario. We quickly learned that conducting experiments outside of a controlled environment poses a plethora of challenges. The communities we were working with are highly cooperative and communal, therefore individual evaluations were perceived as strange and awkward. Furthermore, interrupting people from assisting each other was considered offensive. For most, the scenarios were too abstract and the evaluation too intimidating to properly participate. Consequently many participants actually performed worse than they did during the prior training sessions and mapping exercises.

\section{Audio feedback}

The second iteration took place in early 2015, when we collaborated with the forestry company CIB to develop a Sapellibased application to improve their local understanding of the mapping process by introducing the ability for Mbendjele community members to be directly involved. For this second iteration of fieldwork, the logging company CIB were our key intermediary. We worked directly with four Mbendjele staff who are responsible for managing the company's social mapping programme. They introduced us to local communities and acted as our research assistants and translators, and also contributed to the design of the decision tree and its icons.

During our month-long field visit, we tried to resolve some of the usability challenges that we identified in our previous expedition. An important issue was the understanding and recognition of the icons. Although these were, in most cases, co-designed with the communities in question, not every person had participated in the initial phase of designing or choosing the icons. Additionally, it is important for participants to be able to understand the meaning of an icon, and of the question posed by the assemblage of icons that makes up a screen, even after long periods of not using the system, when navigating a decision tree for the first time, or having had only minimal training.

To improve recognition and recall of the pictorial icons and the questions posed on each of the screens, we developed and evaluated an audio feedback feature. Research has shown that providing information across different human senses can have an impact on a participant performance [8]. Thus, the audio feature enables descriptions of various items on the display to be played back by the application, to aid the user's understanding of the interface. A similar approach was employed in $[40,41]$, where the researchers used audio clips to assist rural users in India to perform micro-finance transactions. For widely spoken languages, Android offers a textto-speech (TTS) service that produces audio files from textual descriptions. However, for less common languages, such as Lingala ${ }^{1}$ or the hundreds of local Bantu- and Ubangianderived languages such as Mbendjele, speech synthesis is not supported yet. Hence, Sapelli was extended to support audio descriptions, using either TTS or pre-recorded audio files to accompany each of the UI elements. In Sapelli surveys a pictorial decision tree represents a question posed to the user, with a list of available options (i.e.icons) to choose from.

\footnotetext{
${ }^{1}$ a Bantu language spoken throughout the north-western part of the Democratic Republic of the Congo (DRC) and the RoC.
} 
Upon reaching a new screen (i.e. decision tree root or node), an audio file narrates the question and then each icon on the screen are sequentially explained by audio playbacks, while an animation signals which icon is being described. In addition, users could long-click on any item, such as an icon or a navigation element, to listen again to a playback describing the particular item.

\section{Audio feedback evaluation}

During 3 weeks in early 2015, we evaluated the audio feedback feature in 4 different communities (Gbagbali, Kabo, Matoto and Sembola) in northern RoC. The visited settlements were either close to CIB's facilities or deep in the forest, reachable only by $4 \times 4$ journeys over narrow dirt roads or the occasional boat ride.

We had a total number of 48 adult participants (24 males, 24 females), who were selected on a voluntary basis on the day of the experiments. Aged between 18 and 69 years old $(\mathrm{M}=34.5, \mathrm{SD}=12.5)$, the majority of the participants were Mbendjele (98\%), while $2 \%$ were Bangombe. A majority of $50 \%$ of the participants had received no formal education, $38 \%$ had primary school education, $6 \%$ had secondary education, while another $6 \%$ stated they had received some education but could not specify which level they had reached.

The decision tree we used for the trials was a reduced version of the one previously designed for the CAGDF project, further modified after discussions with the social cartography team of CIB. Their modifications related mostly to the structure of the tree, adding and eliminating some categories and making alterations to specific icons which they argued would make the icons more comprehensible for the communities. After finalising the decision tree, we recorded, in the local Mbendjele language, all of the questions and icon descriptions to be used in the audio feedback version. The Sapelli survey was loaded on 8 Samsung Xcover 2 smartphones.

The goal of the experiments was to evaluate participants' $a c$ curacy and recall by providing them with a set of representative scenario tasks to complete using both versions, with and without audio feedback. In the first two communities, Gbagbali and Kabo, participants firstly performed the tasks without audio feedback and then completed the same tasks with audio feedback. To counterbalance the results, we followed the reverse order in the last two communities, Matoto and Sembola.

Our experience from the previous usability experiments in the wild informed us that tasks based on hypothetical scenarios (e.g. "Suppose you are ...") do not work well with participants. Thus, we decided to ask our participants to perform five practical tasks, where they had to collect data for five nearby resources under different top-level categories of the decision tree. All the selected points were valuable resources for the community that they would like to protect against damage from future logging activities. For instance: medicinal trees, the local cemetery, cacao trees etc. In addition, to avoid bystanders helping participants who were struggling to understand or perform the tasks, we deliberately chose five points of interest away from the village but within a short walking distance. We walked in the nearby area prior to the trials and, with the assistance of one or two community members, we selected five appropriate points for the experiments.

On the day of the experiments, we followed the FPIC process, described in the Community Engagement in the Wild section, to introduce the project, the project scope, and our technology. Once people seemed comfortable with the application and the way of "tapping" the icons and moving between screens, we asked them to participate, in pairs, in our task trials. Based on the previous usability experiments, we knew people's preference to work together, since data collection is not an individual task, but a group task, where people can pass the phone to each other and discuss their results. The lead author and two research assistants accompanied the participants during each task. The research assistants were part of the social team of CIB and were facilitating the translation, note-taking and video-recording of the participants' interactions with the devices. At each of the sites, the participants were asked to describe the point of interest in front of them (e.g. a medicinal tree), to ensure that they understood its significance. The participants were each holding a smartphone with Sapelli loaded and were then asked to record the type and location of the point. Their task was to follow the appropriate path of the decision tree until they reached the corresponding leaf icon, followed by a screen where they could confirm the observation. At that point the researcher coded their response as correct or incorrect, while the Sapelli app was also logging all the interactions with the device - such that afterwards we could compare the actual use of the system to the perceived use if necessary. We decided that each participant would have a maximum time of 5 minutes to complete a task, although in the experiments no participants needed more than two minutes to record an observation. Initially participants were asked to "think aloud" and collaborate with each other, but it was soon clear that they were either too nervous, or lacked the vocabulary and contextual understanding, to describe their actions and thus the think aloud technique was abandoned. Similarly, Chetty argues that participants in ICT4D projects often lack the experience to comment and suggest alterations on user interfaces, or to contribute in think aloud exercises [10].

Following completion of the tasks, interviews with the two participants, comprising both structured and semi-structured questions, took place. The interviews were video-recorded for later reference and transcription. During the interviews, the researchers tried to facilitate a discussion on usability of and user satisfaction with the app, and to identify the reasons for some participants' poor performance on certain tasks.

\section{Audio feedback results}

As shown in Table 1, the 48 participants completed 240 tasks without, and 240 tasks with, audio feedback enabled. When using the version without audio assistance they performed 177 successful observations $(73.75 \%)$, while using the version with audio assistance, they performed 185 successful observations (77.08\%). The audio prompt thus seemed to be effective in slightly improving participant accuracy. However performing a paired $t$-test revealed that the mean increase in 
accuracy $(\mathrm{M}=0.16, \mathrm{SD}=1.15)$ was not statistically significant $(t(47)=1, p=0.32)$. Figure 2 shows the accuracy for both versions on relation to the number of correct tasks.

\begin{tabular}{llr}
\hline \multirow{2}{*}{ Success } & Non-audio & $177 \mathbf{( 7 3 . 7 5 \% )}$ \\
& Audio & $185 \mathbf{( 7 7 . 0 8 \% )}$ \\
\hline \multirow{2}{*}{ Failure } & Non-audio & $63 \mathbf{( 2 6 . 2 5 \% )}$ \\
\hline \multirow{2}{*}{ Number of Tasks } & Audio & $55(\mathbf{2 2 . 9 2 \% )}$ \\
\cline { 2 - 2 } & Non-audio & 240 \\
& Audio & 240 \\
\hline
\end{tabular}

Table 1. Audio feedback results

Interestingly, the results indicate that the success rates of decision trees are correlated with the literacy level of the participants and their exposure to technology. In Gbagbali and Kabo, two remote communities with lower literacy levels, the success rate for decision trees, without audio, was 63.3\%. When asked if they had ever used a mobile phone before (feature phone or smartphone), only $8 \%$ of participants replied positively. In Matoto and Sembola, communities with higher education levels, which are located closer to the logging company's camp and which have easier access to technology (33\% of participants claimed that they had used a phone before), the success rate was $84.1 \%$.

However, there was a significant difference in terms of user experience and user satisfaction. During the interviews, 33 out of the 48 participants (69\%) stated that they preferred the version with the audio feedback. The main reason given was that the device was speaking their local language, which they found entertaining and reassuring. For many participants the version with audio prompts had a pedagogic element, as it reminded them of school and provided them with knowledge about the icons and the project. For others, the audio feedback was a good way of verifying what they already knew, and of giving them reassurance that they were selecting the appropriate icons. One interesting case involved an older woman who stated that she liked the audio version because her bad eyesight did not allow her to clearly distinguish the icons. Finally, one participant stated that she loved hearing the question, as this made choosing the right icon easier for her. Thirteen participants $(27 \%)$ stated that they liked both versions and could not decide on one; according to them both were very practical and easy to use. Finally, only two participants preferred the version without audio, the first participant was comfortable enough without audio and stated that he didn't need it, while the second claimed that the audio prompt was very distracting for him, since he already knew the answers.

\section{Exploring physical interfaces}

Even though pictorial interfaces reduce the accessibility barriers that text introduces, still they do not provide a universal solution. This is because many users, especially those who have never had any formal education or who are completely unfamiliar with digital technology, face difficulties using the application that audio prompts cannot assist with. The main

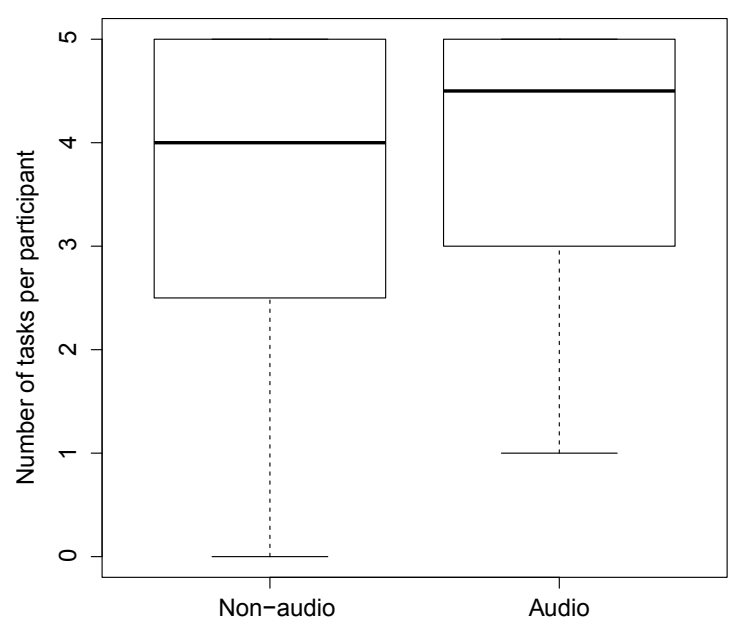

Figure 2. Accuracy performance for non-audio and audio tasks.

barriers identified after close observation and follow-up interviews during the audio feedback trials were: (a) fear of using technology; (b) difficulties in navigation; and (c) inappropriate categorisation.

Many participants, especially those less familiar with technology, expressed a fear or hesitancy around using the smartphones and their touch-screens. In addition, their lack of experience regarding how to tap the display, in combination with fingertip callouses formed as a result of manual labour, restrained participants from easily using the touch-screen and exploring the affordances of the application. The unresponsiveness of the screen led to frustration in some cases, as tapping an icon did not produce any results.

Although the participants did understand the significance of each icon, the interviews showed that some had difficulties with the hierarchical navigation structure and the categorisation implicit in a decision tree. The problem seemed to be twofold; on the one hand participants seemed to not understand the abstract hierarchical structure and the icons used for navigational purposes at the top of the screen. For instance, the function of the icons for navigating back to the previous interface (left arrow), and for cancelling an observation (cross) (Figure 1b) were not clear to all participants and they were rarely used. When asked, one Mbendjele woman said that the left arrow represents medicinal resources, while a Mbendjele man believed it represented " a path leading to the cemetery". The same woman believed that the cancellation cross represented cacao trees, while the man could not explain the significance of the cross. Although these icons were grouped together at the top of the screen and had different look and feel than the "normal" Sapelli icons (different size $\&$ background colour), it was clear that participants did not understand their navigational role and misinterpreted them as ordinary icons that should be used for mapping resources.

On the other hand, the categories themselves and the icons designed to represent them were difficult to interpret. During the recording of the audio clips for the audio feedback experiments it became evident that no generic terms existed in the local language for some of the categories. To compensate 

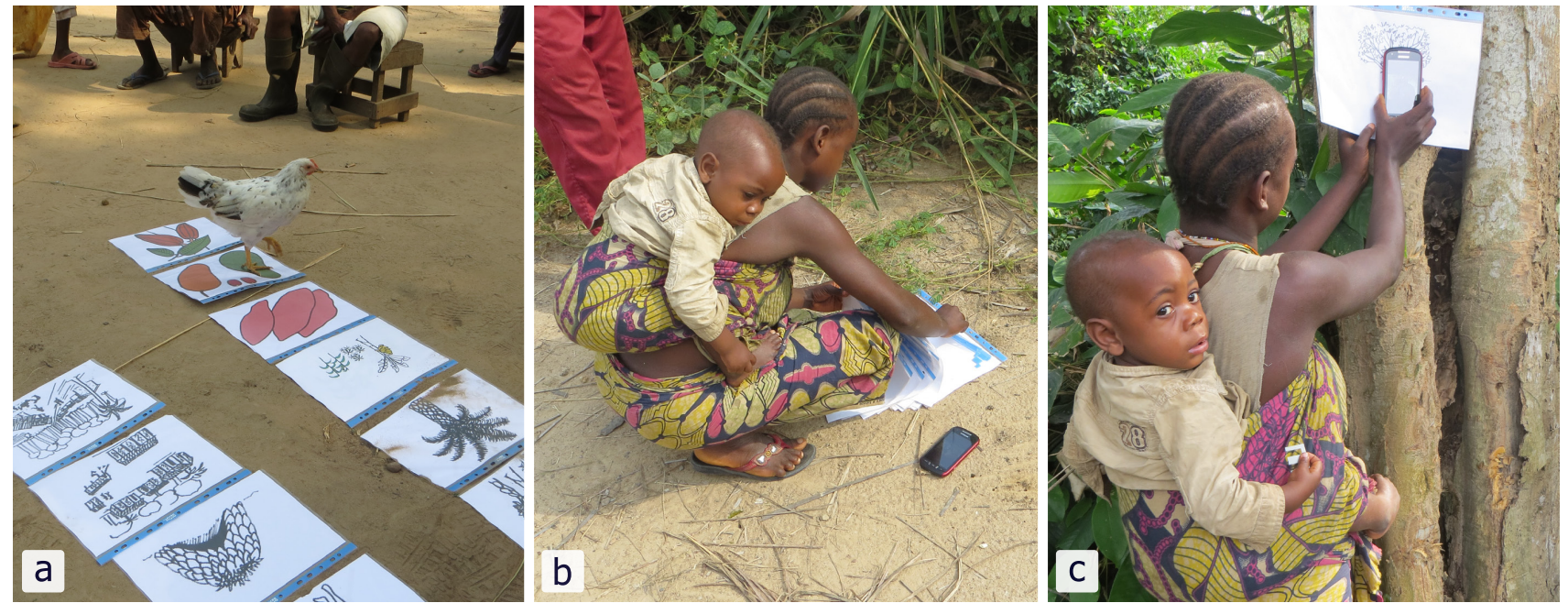

Figure 3. Prototype version of Tap\&Map: a) printed prototype cards b) picking the appropriate card c) mapping a medicinal tree

for that, the audio recordings were often very descriptive and verbose, trying to give examples for the category. On top of that, designing icons for the categories was a major challenge. As explained above, some of the decision tree icons were intended to be interpreted literally while others were meant to represent (intermediate) categories. Yet during the field trials it became clear that category examples were often interpreted literally.

For these reasons, we decided to abandon the planned experiments with animated screen transitions - which we feared would not significantly improve user performance either and instead use our remaining time in the field to explore an alternative interface with a focus on eliminating categorisation and navigational structures and reducing the need for interaction with the device. Recently there has been a growing interest in forms of interaction that combine physical objects and graphical interfaces $[52,26]$. Providing a link with the real world and building on users' knowledge concerning how to interact with physical objects can improve participants' confidence [48]. Hence, we conceptualised a system consisting of two elements to investigate physical interfaces: (a) a series of cards, each with an icon representing a site to be mapped; and (b) a smartphone application. Each card would be equipped with an near field communication (NFC) tag and would then act as a tangible user interface. The application would react when one of the "control" cards is touched on the device. When, for example, a participant wants to record a point of interest, such as a banana tree, he or she: (a) selects the appropriate card from a stack of cards (Figure 3b); (b) touches the card to the phone while standing as close as possible to the site to be mapped (Figure 3c). The device then reads the user's location from the GPS sensor and stores it along with other necessary metadata (such as the selected card, device id, time, etc.). We call this concept Tap\&Map and hypothesised that in comparison to on-screen decision trees it could enable a simpler and more intuitive way for nonliterate participants to map local resources (or collect other kinds of information depending on the project scope).

\section{Tap\&Map evaluation}

Following a rapid prototyping approach, we decided to implement and evaluate a Tap\&Map prototype during our collaboration with CIB. We ended up evaluating the prototype in two communities (Matoto and Sembola) that were located very close to the logging company's facilities.

Thirty-two adult participants ( 15 males, 17 females) took part in the study, selected on a voluntary basis on the days of the experiments. The majority had been present on the previous days during our introduction of Sapelli, but they had not participated in the audio feedback trials. They were aged between 18 and 61 years old $(\mathrm{M}=28.8, \mathrm{SD}=11.4)$ and the majority were Mbendjele (87.5\%), while $12.5 \%$ were Mikaya. With regards to education, $44 \%$ of the participants had no formal education, $47 \%$ had a primary school education, and $9 \%$ had a secondary education.

The printed cards that we used were the same as those used for training purposes during the audio feedback trials (Figure 3a). Since it was impossible to acquire NFC tags in the rainforest, we decided to develop a prototype which imitated the functionality of Tap\&Map. The Android application we developed consisted of three simple UIs: (a) a start screen with a photo of one of our research assistants touching the phone on top of a card to demonstrate how the app works; (b) an interface imitating a GPS waiting screen; and (c) a final screen with a photo of our research assistant giving a "thumbs-up" hand gesture to indicate that a point has been successfully recorded. In order for the application to feel as real as possible, we developed a separate application, installed on another phone, for controlling the Tap\&Map prototype remotely via Bluetooth. The remote application had only three buttons for selecting and changing the displayed UI on the Tap\&Map prototype.

On the day of the experiments, the FPIC process to introduce the project and the icons was followed, as explained above. After introducing the icons using the flashcards, the first author did some demonstrations around the village on how to 
map resources such as trees by selecting the appropriate card, placing the card on the tree trunk and then placing the phone on top of the card. The second author was operating the Bluetooth remote app and changed the displayed UI on Tap\&Map from the initial help screen to the GPS waiting screen, then slightly later to the success screen. Mapping abstract or larger resources, such as the village itself, proved more challenging since there was no single reference point. To demonstrate how to do this, the researcher walked to the centre of the village, selected the village card and held the card in the air while touching it with the phone.

After the demonstration, the procedure of the experiments was very similar to that followed in the audio feedback trials. We used the same five nearby resources and the lead author, along with two research assistants, accompanied two participants to each of the points. Since, working in pairs seemed to have a positive effect and helped participants to relax during our previous trials, we kept the same structure. The research assistants were responsible for video-recording the process, translating and explaining the tasks to the participants. As in the previous trial, the participants were asked to describe the point of interest in front of them. Then they were given a stack of shuffled cards and a mobile phone with Tap\&Map loaded. The participants' task was to map the resources by finding and selecting the appropriate card, placing the card as close as possible to the resource, touching their phone on the card and waiting for the GPS screen and then the success screen to show. The second researcher operated the remote app and switched the UIs (from the waiting screen to the GPS screen and finally to the success screen) to indicate that a complete observation had been performed. At this point the first researcher noted whether the participants had successfully completed the task.

\section{Tap\&Map results}

Over a period of two days, 32 participants completed 160 tasks using Tap\&Map, with a success rate of $97.50 \%$, and failing only on 4 tasks. During the interviews, the participants were very enthusiastic about the Tap\&Map prototype, and unanimously agreed that this version was faster, easier and more comfortable to use compared to Sapelli. All agreed that they had no difficulties in selecting the appropriate icons and performing the tapping exercises. Indicatively, one Mbendjele woman said: "I have never used a mobile phone in my life and I am so happy that it was so easy". A man said that he really liked the version with tapping cards and he volunteered to travel up to the next forest community through the rainforest and collect points with Tap\&Map.

Finally, all four of failed attempts with Tap\&Map occurred when participants tried to map their village. In the scope of the project, participants could map their village and declare whether this was a Pygmies only village or a Bantu village. In all four of instances, participants chose the wrong village icon, instead for example choosing a random icon in general, which suggested that they understood the process but could not distinguish between the icons. This was an indication that the icons symbolising Pygmies and Bantu villages respectively were problematic and had to be redrawn.

\section{DISCUSSION}

This papers contributes to the rich literature of $\mathrm{CSCW}$ and HCI, by providing a methodological approach for introducing and improving communication and collaboration tools between remote communities and stakeholders. Thus, we provide project design and engagement guidelines in similar, challenging environments. Next, the main focus of this paper is on the usability evaluation and improvement of the tools, which could in turn increase the sustainability of the approach. As a result we provide methods for conducting evaluation studies "in the wild". Finally, the novelty of the work described in this paper lies in the conception, development and testing of the interaction modes for data collection (e.g. "Tap\&Map" approach), which permit an entire community, regardless of skills or literacy levels, to create maps of key features of their local environment for use in logging consultations and improve collaboration and communication.

In this section, we discuss our experiences of working with local communities in developing countries, and we introduce their methodological implications for $\mathrm{CSCW}$, HCI and usability studies. We organise the discussion into three subsections: the first is concerned with the design, introduction and engagement procedure for the project; the second concerns the methodology used to evaluate technological solutions; and finally the third subsection explores the interfaces we suggest to facilitate fast and accurate data collection in different contexts.

\section{Project design \& engagement protocol}

In the majority of cases, the introduction of ICTs alone is insufficient for bringing about long-term community engagement and successful development outcomes [61]. Previous studies have estimated that half of attempted projects, conducted under similar circumstances, have been total or partial failures [22, 23]. To overcome challenges such as cultural misunderstandings, raised expectations, misinterpretations of project purpose and scope, unsuitable technological solutions etc., projects should be framed within a well-designed protocol, adapted to local cultural, political and socio-economic demands. We don't claim to have solved all these issues, but we are suggesting a few solutions to target some of them.

Understand local conditions and establish effective partnerships with intermediaries. It is essential to establish good relationships and partnerships with local intermediaries and stakeholders, who are already collaborating closely with local communities. In our projects, such partners are regional authorities, local NGOs and prominent community members, while our team is composed of anthropologists, who conduct ethnographic studies, and technology experts who adapt the ICT solutions based on the local requirements. Understanding local contexts prevents researchers from making assumptions based on their personal beliefs and proficiency. However, at the same time it is important to maintain an awareness that all intermediary stakeholders, including community members, are likely to have their own agendas that may fit to varying extents with the goals of an extreme citizen science project. Understanding and mapping these agendas is vital if the introduction of specially designed software is to have a 
positive and sustainable effect. For example, in the logging case study and although this was a pilot, the amount of collected data overwhelmed the social cartography team of the company who were unsure how to prioritise and use it. In this case, a new set of consultations with the community is necessary until both parties are satisfied. This, once again, indicates that data collection projects are constantly evolving and our tools provide just the means for communication and collaboration.

Manage local expectations and use FPIC procedures to introduce the project, and the technology. As we described in the evaluation section, we are following a detailed FPIC protocol from our first contact with a potential participating community. While introducing the project we try to be honest and open about the potential benefits and risks involved with the project and we ask for consent multiple times during the introduction, the training and the evaluation of the system. It is particularly important to avoid raising expectations, as the processes of technology design/prototyping and technology deployment are separate issues, and deployment may not necessarily result from the research phase. Again, this is highly dependent on the local context, and particularly on local intermediary interest in and capacity to carry on a long-term project after the pilot phase. In the case of the two iterations of software testing described in this paper, CAGDF is currently seeking funding for a wider deployment of Sapelli in their monitoring work, while CIB is now introducing Sapelli into its social mapping work on a pilot basis.

Apply inclusive, participatory design. Our results further support the idea that there is no universal, or "one-size-fits-all", solution and ICT-related initiatives should be adapted to local contexts and needs $[14,61]$. This requires an inclusive and bottom-up collaboration with the participating communities in order to identify the most suitable solutions and improve the solutions based on the communities' input, needs and abilities. It is also an ongoing process, as communities will be more able to provide input as their familiarity with the technology increases. Following the second iteration of usability experiments, we have continued to modify the decision tree structure and icons for the CIB project based on continued community feedback.

Identify and capitalise on "talent". In every community that we worked with, there were participants that grasped the procedure faster than the others and were quicker to develop a relative proficiency in using the tools. We plan to develop peer-to-peer training strategies to encourage these proficient users to transfer their expertise to their peers, and act as local "infomediaries" [61].

\section{Evaluation methodology}

As we briefly described in the above, conducting usability experiments outside of the controlled environment of a lab introduces significant challenges, such as cultural differences, communication difficulties and time constraints.

Establish a strict protocol with research assistants and isolate participants. A key identified issue was the lack of cultural understanding with regard to being evaluated or tested one- by-one. The participating communities were highly cooperative and communal, thus performing individual evaluations was perceived as strange and awkward. Consequently bystanders, and even translators, would often help participants when they struggled to understand or perform the tasks in question, and stopping people from assisting was considered offensive. In our last field trip, we sought to remedy this issue by establishing a strict protocol with our research assistants and by conducting the evaluations during a walk in the nearby forest to separate the participants from the rest of the community. However, instead of working one-to-one, we decided to work with groups of two participants. Following a flexible approach, we had to adjust our expectations due to the way the community prefers to work together, and data collection is not an individual task but a group activity.

Design real-life, practical tasks. A key challenge was the design of the tasks for the evaluation trials. During our first field trip, we introduced scenario tasks to the participants in the form of short, hypothetical stories. However, such scenariobased tasks did not translate well from a local cultural perspective; participants were often unsure what actions were required of them and needed to be talked through the steps of each task explicitly. In our second field trip, we tried to target this issue by engaging participants in real-life, practical tasks, like mapping nearby resources.

Allow room for flexibility. Communication difficulties and finding good translators is another important challenge. We often had to go through multiple steps of translation (e.g. English $>$ French $>$ Lingala $>$ Mbendjele), with the potential for meaning to be lost, changed or added. Next, time constraints is an important factor, due to travelling we generally had limited time to spend in each place. Participants usually also had limited time themselves, due to other obligations (e.g. tending to their fields). Good planning, time flexibility and establishing a detailed protocol with the CIB research assistants helped resolve these issues to some degree.

In terms of usability evaluation methodologies, our observations support the idea that due to lack of experience and relevant vocabulary, methods such as "think aloud" or asking for feedback on an interface are not applicable [10, 49]. Alternatively, we advocate for a mixture of ethnographic approaches and observations, structured and semi-structured interviews and informal conversations.

\section{Interfaces for data collection}

In terms of usability and user interaction, in this study we are proposing three interfaces to overcome the literacy barrier and enable forest community members to engage in participatory monitoring activities: pictorial decision trees; decision trees with audio feedback; and physical interfaces.

Pictorial decision trees constitute a fast and effective method for navigating through classification structures. Each decision tree represents one unique question, and chaining multiple trees allows participants to quickly answer a full survey. In addition, many resent studies highlight the importance of text-free interfaces when designing for non-literate or illiterate participants $[6,18,19,27,29,33,39$, 53, 63]. However, 
as shown in our results, decision trees are not a universal solution. Community success rates vary from $60 \%$ to $85 \%$ and seem correlated with the average level of education. For instance, in our experiments in Gbagbali and Kabo, two remote communities with lower average levels of education, the success rate of working with decision trees was $63.3 \%$; while in Matoto and Sembola, which are communities with higher average levels of education, participants achieved success rates of $84.1 \%$.

The second interface we suggest is complementing decision trees with audio feedback that explains through pre-recorded audio clips, in the local language, the meaning of each icon to the participant. While research in similar context suggests that audio had a significant improvement in accuracy [40, 41], as shown by our results, the audio feedback feature resulted in a slight, but not statistically significant, improvement in participant's average accuracy. What is interesting is that the majority of the participants preferred to use the audio feedback version, even though it didn't significantly improve their performance. Speaking their own language, the device was able to entertain, reassure and verify the participant's choices, which we believe could lead to higher engagement with the project. We therefore advocate for the use of audio interfaces, especially in the early stages of a project and during training sessions, to engage with the community and potentially reduce their learning curve.

Finally, we suggest the use of physical, tangible interfaces in scenarios where users have minimal or no formal education or familiarity with technology. As our results indicate, physical interfaces provide comprehensible links between participants' knowledge, the real world and digital interfaces. This leads to higher levels of confidence and performance, and enhances the overall user experience.

\section{Limitations}

Although, this paper demonstrates the technical feasibility of the approach, the adoption depends on the agendas and priorities of local stakeholders and policy makers. As noted, the case studies described in this paper were both pilot studies with no assurance for further deployments. CAGDF is currently seeking funding for a wider deployment of Sapelli in their monitoring work and CIB is introducing Sapelli into its social mapping work on a pilot basis. Additionally, our approach requires technology (i.e. smartphones) that is not present in the communities, thus local NGOs or other interested parties (e.g. logging company) have to invest in providing and maintaining that technology, which introduces financial and logistical challenges. As a result, it is our responsibility to manage local expectations and clearly explain the limitations at every stage of our research.

In terms of our tools, all the suggested solutions have shortcomings and limitations. The options offered for data collection, in all of the interfaces, are strictly predefined during the design phase of the project or survey. In other words, users are restricted to the icons offered in a Sapelli survey, or by the NFC cards they are given for use with Tap\&Map. This limitation means that a monitoring project should be regularly followed-up and continually adapted to match all the stakeholders' needs and requirements. Next, the questions to be answered are not directly apparent, but are implied to the user by the options provided as answers. Audio feedback can tackle this limitation by speaking out loud the question to the participants. This could be very beneficial during training sessions and the first stages of a project, until participants feel confident enough with the questions. Although, we demonstrated that using different interaction modes, we improved the usability, the accuracy and the user satisfaction of our tools, there is still no guarantee that the collected data is entirely accurate. As a result, mechanisms to verify and improve the validity of the data should be explored. For instance, one strategy could be of multiple participants collecting the same information and cross-verifying the results.

Lastly, the UIs we have evaluated target simplicity and do not offer any direct feedback to participants in terms of the data collected, i.e. number of points, etc. In the future we will investigate tools and methodologies to enable visualisation, analysis and editing of spatio-temporal data in ways intelligible to local communities.

\section{CONCLUSIONS \& FUTURE WORK}

The present study contributes to the blossoming research area of creating ICT systems to support community-based mapping and monitoring in remote areas and the methodological challenges that this poses for CSCW and HCI. We have presented our work with marginalised groups, such as indigenous forest communities in the RoC, to enable them to use scientifically acceptable methods to collect local environmental data and collaboratively participate in decision-making processes.

We have introduced the key elements of our methodological approach for engaging with participants and introducing the project and the technology to local communities, who have often received little or no formal education or exposure to technology. We have also provided an in-depth look at our methodology for conducting usability experiments outside of the controlled and "safe" environment of the lab.

In terms of user interaction, we are proposing three different interfaces to enable local people to participate in data collection schemes and monitoring activities. We suggest pictorial decision trees as an effective method for semi-literate participants, without and with audio feedback for enhancing the user experience. Finally we propose physical interfaces for users with minimal or no formal education for improving their confidence and performance.

In the future we plan to further develop the Tap\&Map prototype and explore methods for integrating its functionality into Sapelli to offer a rich data collection experience, incorporating opportunities for data entry via either physical interfaces, pictorial decision trees, or textual input. The goal is to create an easily adaptable system that could be configured depending on each participant's needs, skills and requirements.

Although our methodologies and the tools we present here are still works in progress, they could provide a concrete base for long-term engagement and successful development outcomes. Apart from the RoC, using the same methodologies 
we are working with communities in the Brazilian Amazon to develop land management tools, while in Namibia our tools will be used by Jul'hoansi communities to monitor and report on illegal invasions of their lands by cattle ranchers.

\section{ACKNOWLEDGEMENTS}

This work has been supported by the Extreme Citizen Science (ExCiteS) grant, funded by the Engineering and Physical Sciences Research Council (EPSRC reference EP/I025278/1). We thank Dr Claire Ellul and Dr Charlene Jennett for providing valuable feedback on earlier drafts. Finally, we thank the anonymous reviewers for their extensive and constructive feedback.

\section{REFERENCES}

1. David M. Aanensen, Derek M. Huntley, Edward J. Feil, Fada'a Al Own, and Brian G. Spratt. 2009. EpiCollect:

Linking Smartphones to Web Applications for Epidemiology, Ecology and Community Data Collection. 4, 9 (Sept. 2009). DOI : http://dx.doi.org/10.1371/journal.pone.0006968

2. Yaw Anokwa, Carl Hartung, Waylon Brunette, Gaetano Borriello, and Adam Lerer. 2009. Open Source Data Collection in the Developing World. Computer 42, 10 (oct 2009), 97-99. DOI : http://dx.doi.org/10.1109/mc.2009.328

3. Shaun Ansell and Jennifer Koenig. 2011. CyberTracker: An integral management tool used by rangers in the Djelk Indigenous Protected Area, central Arnhem Land, Australia. 12, 1 (2011), 13-25. DOI : http: //dx.doi.org/10.1111/j.1442-8903.2011.00575.x

4. Richard P. Bagozzi, Fred D. Davis, and Paul R. Warshaw. 1992. Development and Test of a Theory of Technological Learning and Usage. Human Relations 45, 7 (July 1992), 659-686. DOI : http://dx.doi.org/10.1177/001872679204500702

5. Fikret Berkes. 2012. Sacred Ecology. Routledge, London,UK

6. Anuradha Bhamidipaty and P. Deepak. 2007. SymAB: Symbol-based Address Book for the Semi-literate Mobile User. In Proceedings of the $11^{\text {th }}$ IFIP TC 13 International Conference on Human-computer Interaction (2007) (INTERACT'07). Springer-Verlag, Berlin, Heidelberg, 389-392. http:

//dl.acm.org/citation. cfm?id=1776994.1777041

7. R. Bonney, J. L. Shirk, T. B. Phillips, A. Wiggins, H. L. Ballard, A. J. Miller Rushing, and J. K. Parrish. 2014. Next Steps for Citizen Science. Science 343, 6178 (mar 2014), 1436-1437. DOI :

http://dx.doi.org/10.1126/science. 1251554

8. Stephen A. Brewster, Peter C. Wright, and Alistair D. N. Edwards. 1993. An evaluation of earcons for use in auditory human-computer interfaces. In Proceedings of the SIGCHI conference on Human factors in computing systems - CHI '93 (1993). Association for Computing Machinery (ACM), Amsterdam, Netherlands, 222-227. DOI : http://dx.doi .org/10.1145/169059.169179
9. Beenish M. Chaudry, Kay H. Connelly, Katie A. Siek, and Janet L. Welch. 2012. Mobile Interface Design for Low-literacy Populations. In Proceedings of the $2^{\text {nd }}$ ACM SIGHIT International Health Informatics Symposium (2012) (IHI'12). ACM, 91-100. DOI : http://dx.doi.org/10.1145/2110363.2110377

10. Marshini Chetty and Rebecca Grinter Chetty. 2007. HCI4D: HCI challenges in the global south. In $\mathrm{CHI}$ '07 extended abstracts on Human factors in computing systems - CHI '07 (2007). Association for Computing Machinery (ACM), San Jose, California, USA, 2327-2332. DOI : http://dx.doi.org/10.1145/1240866.1241002

11. Jan Chipchase. 2006. How Do You Manage Your Contacts if You Can't Read or Write? interactions 13, 6 (Nov. 2006), 16-17. DOI : http://dx.doi.org/10.1145/1167948.1167966

12. Cybertracker. 2015. Cybertracker. (2015). http: //cybertracker.org/

13. F. D. Davis. 1989. Perceived usefulness, perceived ease of use, and user acceptance of information technology. MIS quarterly 13, 3 (1989), 319-340. DOI : http://dx.doi.org/10.2307/249008

14. Bob Day and Peter Greenwood. 2009. Information and communication technologies for rural development. In ICT4D: Information and Communication Technology for Development (1 ed.), Tim Unwin (Ed.). Cambridge University Press, Cambridge, UK, 321-359.

15. Robert Douman. 2006. Distributed Data Collection Extensions to the CyberTracker mobile application. Master's thesis.

16. Paul R. Ehrlich and Anne H. Ehrlich. 2012. Solving the human predicament. International Journal of Environmental Studies 69, 4 (aug 2012), 557-565. DOI : http://dx.doi.org/10.1080/00207233.2012.693281

17. E. Ens. 2012. Monitoring outcomes of environmental service provision in low socio-economic indigenous Australia using innovative CyberTracker Technology. 10, 1 (2012), 42-52. DOI : http://dx.doi.org/10.4103/0972-4923.92194

18. Kaushik Ghosh, Tapan S Parikh, and Apala Lahiri Chavan. 2003. Design considerations for a financial management system for rural, semi-literate users. In CHI'03 Extended Abstracts on Human Factors in Computing Systems (2003). ACM, 824-825.

19. Sally Grisedale, Mike Graves, and Alexander Grünsteidl. 1997. Designing a graphical user interface for healthcare workers in rural India. In Proceedings of the SIGCHI conference on Human factors in computing systems - CHI '97 (1997). ACM Press, 471-478. DOI : http://dx.doi.org/10.1145/258549.258869

20. Aditi Sharma Grover, Madelaine Plauché, Etienne Barnard, and Christiaan Kuun. 2009. HIV health information access using spoken dialogue systems: 
touchtone vs. speech. In ICTD'09: Proceedings of the $3^{\text {rd }}$ international conference on Information and communication technologies and development (2009) (ICTD'09). IEEE Press, 95-107. http:

$/ / \mathrm{dl}$.acm.org/citation. cfm?id=1812530.1812541

21. Carl Hartung, Adam Lerer, Yaw Anokwa, Clint Tseng, Waylon Brunette, and Gaetano Borriello. 2010. Open Data Kit: Tools to Build Information Services for Developing Regions. In Proceedings of the $4^{\text {th }}$ ACM/IEEE International Conference on Information and Communication Technologies and Development (2010) (ICTD '10). ACM, New York, NY, USA, Article 18, 12 pages. DOI :

http://dx.doi.org/10.1145/2369220.2369236

22. Richard Heeks. 2002. Information Systems and Developing Countries: Failure, Success, and Local Improvisations. The Information Society 18, 2 (mar 2002), 101-112. DOI :

http://dx.doi.org/10.1080/01972240290075039

23. Richard Heeks. 2010. Do information and communication technologies (ICTs) contribute to development? Journal of International Development 22, 5 (jun 2010), 625-640. DOI : http://dx.doi.org/10.1002/jid.1716

24. Henry P. Huntington. 2011. Arctic science: The local perspective. Nature 478, 7368 (oct 2011), 182-183. DOI : http://dx.doi.org/10.1038/478182a

25. Lilly Irani, Janet Vertesi, Paul Dourish, Kavita Philip, and Rebecca E. Grinter. 2010. Postcolonial computing. In Proceedings of the 28th international conference on Human factors in computing systems - CHI '10 (2010). Association for Computing Machinery (ACM). DOI : http://dx.doi.org/10.1145/1753326.1753522

26. Kasper Løvborg Jensen. 2012. Sensible smartphones for Southern Africa. interactions 19, 4 (July 2012), 66. DOI : http://dx.doi.org/10.1145/2212877.2212893

27. Anirudha Joshi, Nikhil Welankar, Naveen BL, Kirti Kanitkar, and Riyaj Sheikh. 2008. Rangoli: A Visual Phonebook for Low-literate Users. In MobileHCI '08: Proceedings of the $10^{\text {th }}$ International Conference on Human Computer Interaction with Mobile Devices and Services (2008). ACM Press, 217-223. DOI : http://dx.doi.org/10.1145/1409240.1409264

28. Neesha Kodagoda, B.L. William Wong, Chris Rooney, and Nawaz Khan. 2012. Interactive Visualization for Low Literacy Users: From Lessons Learnt to Design. In Proceedings of the SIGCHI Conference on Human Factors in Computing Systems (2012) (CHI'12). ACM, 1159-1168. DOI :

http://dx.doi.org/10.1145/2207676.2208565

29. Zereh Lalji and Judith Good. 2008. Designing new technologies for illiterate populations: A study in mobile phone interface design. 20, 6 (2008), 574-586. DOI : http://dx.doi.org/10.1016/j.intcom.2008.09.002
30. Nicholas Lane, Emiliano Miluzzo, Hong Lu, Daniel Peebles, Tanzeem Choudhury, and Andrew Campbell. 2010. A survey of mobile phone sensing. 48, 9 (Sept. 2010), 140-150. DOI : http://dx.doi.org/10.1109/mcom.2010.5560598

31. Samuel Lefever, Michael Dal, and Ásrún Matthíasdóttir. 2007. Online data collection in academic research: advantages and limitations. 38, 4 (2007), 574-582. DOI : http: //dx.doi.org/10.1111/j.1467-8535.2006.00638.x

32. Jerome Lewis. 2012. Technological leap-frogging in the Congo Basin. Pygmies and geographic positioning systems in Central Africa: What has happened and where is it going? African Study Monographs 43, March (March 2012), 15-44. http://repository . kulib. kyoto-u.ac.jp/dspace/handle/2433/153065

33. Jerome Lewis and John Nelson. 2006. Logging in the Congo Basin: What Hope for Indigenous Peoples' Resources and their Environments? International Work Group for Indigenous Affairs (IWGIA) 4/06 (Feb. 2006), 8-15. http://www.iwgia.org/publications/ search-pubs?publication_id $=45$

34. Jerome Lewis and Teodyl Nkuintchua. 2012. Accessible technologies and FPIC: independent monitoring with forest communities in Cameroon. Participatory Learning and Action Jun, 65 (2012), 151-165.

35. Matthias Stevens, Michalis Vitos, Julia Altenbuchner, Gillian Conquest, Jerome Lewis, and Muki Haklay. 2014. Taking Participatory Citizen Science to Extremes. IEEE Pervasive Computing 13, 2 (April-June 2014), 20-29. DOI : http://dx.doi.org/10.1109/MPRV.2014.37

36. Indrani Medhi, S.N. Nagasena Gautama, and Kentaro Toyama. 2009. A Comparison of Mobile Money-transfer UIs for Non-literate and Semi-literate Users. In Proceedings of the SIGCHI Conference on Human Factors in Computing Systems (2009) (CHI '09). ACM, New York, NY, USA, 1741-1750. DOI : http://dx.doi.org/10.1145/1518701.1518970

37. Indrani Medhi, Meera Lakshmanan, Kentaro Toyama, and Edward Cutrell. 2013. Some evidence for the impact of limited education on hierarchical user interface navigation. In Proceedings of the SIGCHI Conference on Human Factors in Computing Systems - CHI '13 (2013). Association for Computing Machinery (ACM), Paris, France, 2813-2822. DOI :

http://dx.doi.org/10.1145/2470654.2481390

38. Indrani Medhi, Aman Sagar, and Kentaro Toyama. 2006. Text-Free User Interfaces for Illiterate and Semi-Literate Users. In Information and Communication Technologies and Development, 2006. ICTD '06. International Conference on (2006). 72-82. DOI : http://dx.doi.org/10.1109/ICTD.2006.301841

39. Tapan Parikh, Kaushik Ghosh, and Apala Chavan. 2003. Design studies for a financial management system for 
micro-credit groups in rural india. In ACM SIGCAPH Computers and the Physically Handicapped (2003). ACM, 15-22.

40. Tapan Parikh, Paul Javid, K. Sasikumar, Kaushik Ghosh, and Kentaro Toyama. 2006. Mobile phones and paper documents: evaluating a new approach for capturing microfinance data in rural India. In Proceedings of the SIGCHI conference on Human Factors in computing systems - CHI 'O6 (2006). Association for Computing Machinery (ACM). DOI : http://dx.doi.org/10.1145/1124772.1124857

41. Tapan Parikh and Edward Lazowska. 2006. Designing an Architecture for Delivering Mobile Information Services to the Rural Developing World. In Proceedings of the $15^{\text {th }}$ International Conference on World Wide Web (2006) (WWW'06). ACM, 791-800. DOI : http://dx.doi.org/10.1145/1135777.1135897

42. Cynthia Sims Parr, Tricia Jones, Nancy Butler Songer, and Ann Arbor. 2002. CyberTracker in BioKIDS: Customization of a PDA-based scientific data collection application for inquiry learning. (2002), 1-8.

43. Neil Patel, Deepti Chittamuru, Anupam Jain, Paresh Dave, and Tapan Parikh. 2010. Avaaj Otalo. In Proceedings of the $28^{\text {th }}$ international conference on Human factors in computing systems - CHI'10 (2010). Association for Computing Machinery (ACM), 733. DOI : http://dx.doi.org/10.1145/1753326.1753434

44. Madelaine Plauché, Udhyakumar Nallasamy, Joyojeet Pal, Chuck Wooters, and Divya Ramachandran. 2006. Speech Recognition for Illiterate Access to Information and Technology. In 2006 International Conference on Information and Communication Technologies and Development (2006). Institute of Electrical \& Electronics Engineers (IEEE), 83-92. DOI : http://dx.doi.org/10.1109/ictd.2006.301842

45. Hardy Pundt. 2002. Field Data Collection with Mobile GIS: Dependencies Between Semantics and Data Quality. 6, 4 (2002), 363-380. DOI : http://dx.doi.org/10.1023/A:1020805511054

46. Peter Reason and Hilary Bradbury. 2008. The SAGE Handbook of Action Research: Participative Inquiry and Practice. SAGE Publications Ltd.

https://uk. sagepub.com/en-gb/eur/ the-sage-handbook-of-action-research/ book228865

47. Thomas Reitmaier, Nicola J. Bidwell, and Gary Marsden. 2010. Field testing mobile digital storytelling software in rural Kenya. In Proceedings of the 12th international conference on Human computer interaction with mobile devices and services MobileHCI '10 (2010). Association for Computing Machinery (ACM). DOI : http://dx.doi.org/10.1145/1851600.1851649

48. Jun Rekimoto, Brygg Ullmer, and Haruo Oba. 2001. DataTiles: a modular platform for mixed physical and graphical interactions. In Proceedings of the SIGCHI conference on Human factors in computing systems CHI '01 (2001). Association for Computing Machinery (ACM), Seattle, USA, 269-276. DOI : http://dx.doi.org/10.1145/365024.365115

49. Kasper Rodil, Søren Eskildsen, Ann Morrison, and Matthias Rehm. 2012. Unlocking good design does not rely on designers alone. In Proceedings of the Annual ACM Conference on Human Factors in Computing Systems - CHI '12 (2012). Association for Computing Machinery (ACM).

50. Yvonne Rogers. 2011. Interaction Design Gone Wild: Striving for Wild Theory. interactions 18, 4 (July 2011), 58-62. DOI :

http://dx.doi.org/10.1145/1978822.1978834

51. Mary Beth Rosson and John M. Carroll. 2001. Usability Engineering: Scenario-Based Development of Human-Computer Interaction (Interactive Technologies). Morgan Kaufmann.

52. Orit Shaer. 2009. Tangible User Interfaces: Past, Present, and Future Directions. FNT in Human-Computer Interaction 3, 1-2 (2009), 1-137. DOI : http://dx.doi .org/10.1561/1100000026

53. H. Shakeel and M.L. Best. 2002. Community knowledge sharing: an Internet application to support communications across literacy levels. In IEEE 2002 International Symposium on Technology and Society (ISTAS'02). Social Implications of Information and Communication Technology. (2002). Institute of Electrical \& Electronics Engineers (IEEE). DOI : http://dx.doi.org/10.1109/istas.2002.1013793

54. Jahanzeb Sherwani, Sooraj Palijo, Sarwat Mirza, Tanveer Ahmed, Nosheen Ali, and Roni Rosenfeld. 2009. Speech vs. touch-tone: Telephony interfaces for information access by low literate users. In 2009 International Conference on Information and Communication Technologies and Development (ICTD) (2009). Institute of Electrical \& Electronics Engineers (IEEE). DOI : http://dx.doi.org/10.1109/ictd.2009.5426682

55. Laura Spinney. 1998. Keeping Track. 2158 (1998), 25.

56. Jeffrey M. Stanton. 1998. An Empirical Assessment of Data Collection Using the Internet. 51, 3 (Sept. 1998), 709-725. DOI : http:

//dx.doi.org/10.1111/j.1744-6570.1998.tb00259.x

57. Matthias Stevens, Michalis Vitos, Julia Altenbuchner, Gillian Conquest, Jerome Lewis, and Muki Haklay. 2013a. Introducing Sapelli: a mobile data collection platform for non-literate users. In Proceedings of the 4th Annual Symposium on Computing for Development (2013) (ACM DEV-4'13). Association for Computing Machinery (ACM), Cape Town, South Africa, Article No.: 17. DOI : http://dx.doi.org/10.1145/2537052.2537069 
58. Matthias Stevens, Michalis Vitos, Jerome Lewis, and Muki Haklay. 2013b. Participatory monitoring of poaching in the Congo basin. In Proceedings of the GIS Research UK (GISRUK) (2013). http:

//www.geos.ed.ac.uk/ gisteac/proceedingsonline/ GISRUK2013/gisruk2013_submission_12.pdf

59. Kamala Thriemer, Benedikt Ley, Shaali M Ame, Mahesh K Puri, Ramadhan Hashim, Na Yoon Chang, Luluwa a Salim, R Leon Ochiai, Thomas F Wierzba, John D Clemens, Lorenz von Seidlein, Jaqueline L Deen, Said M Ali, and Mohammad Ali. 2012. Replacing paper data collection forms with electronic data entry in the field: findings from a study of community-acquired bloodstream infections in Pemba, Zanzibar. 5, 1 (Jan. 2012), 113. DOI :

http://dx.doi.org/10.1186/1756-0500-5-113

60. UIS. 2013. International Literacy Data 2013. (2013). http://www. uis. unesco.org/literacy/Pages/ data-release-map-2013.aspx

61. Tim Unwin. 2009. ICT4D: Information and Communication Technology for Development. Cambridge University Press, Cambridge, UK.

62. Rajesh Veeraraghavan, Naga Yasodhar, and Kentaro Toyama. 2007. Warana Unwired: Replacing PCs with mobile phones in a rural sugarcane cooperative. In 2007 International Conference on Information and Communication Technologies and Development (2007). IEEE, 1-10. DOI :

http://dx.doi.org/10.1109/ICTD.2007.4937395
63. Michalis Vitos, Matthias Stevens, Jerome Lewis, and Muki Haklay. 2012. Community mapping by non-literate citizen scientists in the rainforest. Bulletin of the Society of Cartographers 46, 1-2 (2012), 3-11.

64. Michalis Vitos, Matthias Stevens, Jerome Lewis, and Muki Haklay. 2013. Making Local Knowledge Matter: Supporting Non-literate People to Monitor Poaching in Congo. In Proceedings of the 3rd ACM Symposium on Computing for Development (2013) (ACM DEV'13). ACM, Bangalore, India, 1:1-1:10. DOI : http://dx.doi.org/10.1145/2442882.2442884

65. Volker Wulf, Kaoru Misaki, Meryem Atam, David Randall, and Markus Rohde. 2013. 'On the ground' in Sidi Bouzid: investigating social media use during the tunisian revolution. In Proceedings of the 2013 conference on Computer supported cooperative work $C S C W^{\prime} 13$ (2013). Association for Computing Machinery (ACM). DOI :

http://dx.doi.org/10.1145/2441776.2441935 